\title{
Convex Structure in Generalized Fuzzy Metric Spaces
}

\author{
V. Vinoba, V. Pazhani, and M. Jeyaraman
}

\begin{abstract}
In this paper, we introduce the concept of convex structure in generalized fuzzy metric spaces and proved common fixed point theorems for a pair of self-mappings under sufficient contractive type conditions.
\end{abstract}

Key words - Contraction mapping, Convex structure, Generalized fuzzy metric space, Metric space.

\section{INTRODUCTION}

In Mathematics, the concept of fuzzy set was introduced by Zadeh [12]. It is a new way to represent vagueness in our daily life. In 1975, Kramosil and Michalek [4] introduced the concept of fuzzy metric spaces which opened a new way for further development of analysis in such spaces. George and Veeramani [1] modified the concept of fuzzy metric space. After that, several fixed point theorems proved in fuzzy metric spaces. In this paper, we introduce the concept of convex structure in generalized fuzzy metric spaces and obtain fixed point and common fixed point theorems for a pair of self-mappings under sufficient contractive type conditions with convex structure.

\section{PRELIMINARIES}

\section{A. Definition $_{b} 2.1$}

Let $(\mathrm{X}, G, *)$ be a $G$ - fuzzy metric space and $\mathrm{I}=[0,1]$. A continuous mapping $\triangle: \mathrm{X} \times \mathrm{X} \times \mathrm{I} \rightarrow \mathrm{X}$ is said to be a convex structure on $\mathrm{X}$ if for each $(\mathrm{x}, \mathrm{y}, k) \in \mathrm{X} \mathrm{x} X \mathrm{x}$ I and $\mathrm{u} \in \mathrm{X}, G(\mathrm{u}, \triangle(\mathrm{x}, \mathrm{y}, k), \triangle(\mathrm{x}, \mathrm{y}, k), \mathrm{t}) \geq k G(\mathrm{u}, \mathrm{x}, \mathrm{x}, \mathrm{t}) \mathrm{b}+(1-$ k) $G(\mathrm{u}, \mathrm{y}, \mathrm{y}, \mathrm{t})$.

A space $\mathrm{X}$ together with a convex structure $\mathrm{R}$ is called a fuzzy convex metric space.

\section{B. Definition 2.2}

A sequence $\left\{\mathrm{x}_{\mathrm{n}}\right\}$ in a generalized fuzzy convex metric space $(\mathrm{X}, G, *)$ is said to converge to $\mathrm{x} \in \mathrm{X}$ if $\lim _{\mathrm{n} \rightarrow \infty} G\left(\mathrm{x}, \mathrm{x}, \mathrm{x}_{\mathrm{n}}\right.$, $\mathrm{t})=1$, for all $\mathrm{t}>0$

\section{Definition 2.3}

Let $(\mathrm{X}, G, *)$ be a generalized fuzzy convex metric space. A sequence $\left\{x_{n}\right\}$ is called Cauchy sequence if $\lim _{n \rightarrow \infty} G\left(x_{n+p}\right.$, $\left.\mathrm{x}_{\mathrm{n}+\mathrm{p}}, \mathrm{x}_{\mathrm{n}}, \mathrm{t}\right)=1$, for all $\mathrm{t}>0$ and $\mathrm{p}>0$.

Published on August 16, 2021

V. Vinoba, Kunthavai Naachiyaar Govt. Arts College for Women(A), Thanjavur, Tamilnadu, Bharadhidhasan University, Thiruchirappalli, India.

V. Pazhani, R. D. Govt. Arts College, Sivagangai, Bharadhidhasan University, Thiruchirappalli, Tamil Nadu, India.

(e-mail: pazhanin ${ }^{\circledR}$ yahoo.com)

\section{Definition 2.4}

A generalized fuzzy convex metric space $(\mathrm{X}, G, *)$ is said to be complete if every Cauchy sequence is convergent.

\section{MAIN RESULTS}

\section{A. Theorem 3.1}

Let $(\mathrm{X}, G, *)$ be a generalized fuzzy convex metric space with $\triangle$ convex structure and let $\Gamma: \mathrm{X} \rightarrow \mathrm{X}$ be self mappings satisfying, $G(\Gamma \mathrm{x}, \Gamma \mathrm{y}, \Gamma \mathrm{y}, k \mathrm{t}) \geq G(\mathrm{x}, \Gamma \mathrm{y}, \Gamma \mathrm{y}, \mathrm{t}) * G(\Gamma \mathrm{x}, \mathrm{y}, \mathrm{y}, \mathrm{t})$, for all $\mathrm{x}, \mathrm{y} \in \mathrm{X}$ and $0<k<1$. Suppose that $\left\{\mathrm{x}_{\mathrm{n}}\right\}$ associated with $\Gamma$ is defined by:

$\mathrm{X}_{0} \in \mathrm{X}$

$\mathrm{x}_{\mathrm{n}+1}=\Delta\left(\Gamma \mathrm{y}_{\mathrm{n}}, \mathrm{x}_{\mathrm{n}}, \alpha_{\mathrm{n}}\right), \mathrm{n}=0,1,2 \ldots$

$\mathrm{y}_{\mathrm{n}}=\Delta\left(\Gamma \mathrm{x}_{\mathrm{n}}, \mathrm{x}_{\mathrm{n}}, \beta_{\mathrm{n}}\right), \mathrm{n}=0,1,2 \ldots$

where $0 \leq \alpha_{\mathrm{n}}, \beta_{\mathrm{n}} \leq 1$ and $\beta_{\mathrm{n}}$ is bounded away from zero. If $\left\{\mathrm{x}_{\mathrm{n}}\right\}$ converges to some point $\mathrm{x} \in \mathrm{X}$ then $\mathrm{x}$ is the fixed point of $\Gamma$.

\section{B. Proof}

Suppose $\mathrm{x}_{\mathrm{n}} \rightarrow \mathrm{x}$.

$G\left(\mathrm{x}_{\mathrm{n}}, \mathrm{x}_{\mathrm{n}+1}, \mathrm{x}_{\mathrm{n}+1}, \mathrm{t}\right)=G\left(\mathrm{x}_{\mathrm{n}}, \triangle\left(\Gamma \mathrm{y}_{\mathrm{n}}, \mathrm{x}_{\mathrm{n}}, \alpha_{\mathrm{n}}\right), \Delta\left(\Gamma \mathrm{y}_{\mathrm{n}}, \mathrm{x}_{\mathrm{n}}, \alpha_{\mathrm{n}}\right), \mathrm{t}\right)$

$\geq \alpha_{\mathrm{n}} G\left(\mathrm{x}_{\mathrm{n}}, \Gamma \mathrm{y}_{\mathrm{n}}, \Gamma \mathrm{y}_{\mathrm{n}}, \mathrm{t}\right)+\left(1-\alpha_{\mathrm{n}}\right) G\left(\mathrm{x}_{\mathrm{n}}, \mathrm{x}_{\mathrm{n}}, \mathrm{x}_{\mathrm{n}}, \mathrm{t}\right)$

$\geq \alpha_{\mathrm{n}} G\left(\mathrm{x}_{\mathrm{n}}, \Gamma \mathrm{y}_{\mathrm{n}}, \Gamma \mathrm{y}_{\mathrm{n}}, \mathrm{t}\right)+\left(1-\alpha_{\mathrm{n}}\right)$

Therefore $G\left(\mathrm{x}_{\mathrm{n}}, \Gamma \mathrm{y}_{\mathrm{n}}, \Gamma \mathrm{y}_{\mathrm{n}}, \mathrm{t}\right)=1$ and hence $\Gamma \mathrm{y}_{\mathrm{n}} \rightarrow \mathrm{x}$.

$$
G\left(\Gamma \mathrm{x}_{\mathrm{n}}, \mathrm{y}_{\mathrm{n}}, \mathrm{y}_{\mathrm{n}}, \mathrm{t}\right)=G\left(\Gamma \mathrm{x}_{\mathrm{n}}, \triangle\left(\Gamma \mathrm{x}_{\mathrm{n}}, \mathrm{x}_{\mathrm{n}}, \beta_{\mathrm{n}}\right), \triangle\left(\Gamma \mathrm{x}_{\mathrm{n}}, \mathrm{x}_{\mathrm{n}}, \beta_{\mathrm{n}}\right), \mathrm{t}\right)
$$

$\geq \beta_{\mathrm{n}} G\left(\Gamma \mathrm{x}_{\mathrm{n}}, \Gamma \mathrm{x}_{\mathrm{n}}, \Gamma \mathrm{x}_{\mathrm{n}}, \mathrm{t}\right)+\left(1-\beta_{\mathrm{n}}\right) G\left(\Gamma \mathrm{x}_{\mathrm{n}}, \mathrm{x}_{\mathrm{n}}, \mathrm{x}_{\mathrm{n}}, \mathrm{t}\right)$

$\geq \beta_{\mathrm{n}}+\left(1-\beta_{\mathrm{n}}\right) G\left(\Gamma \mathrm{x}_{\mathrm{n}}, \mathrm{x}_{\mathrm{n}}, \mathrm{x}_{\mathrm{n}}, \mathrm{t}\right)$

$G\left(\Gamma \mathrm{x}_{\mathrm{n}}, \Gamma \mathrm{y}_{\mathrm{n}}, \Gamma \mathrm{y}_{\mathrm{n}}, k \mathrm{t}\right) \geq G\left(\mathrm{x}_{\mathrm{n}}, \Gamma \mathrm{y}_{\mathrm{n}}, \Gamma \mathrm{y}_{\mathrm{n}}, \mathrm{t}\right) * G\left(\Gamma \mathrm{x}_{\mathrm{n}}, \mathrm{y}_{\mathrm{n}}, \mathrm{y}_{\mathrm{n}}, \mathrm{t}\right)$

$\geq 1 *\left[\beta_{\mathrm{n}}+\left(1-\beta_{\mathrm{n}}\right) G\left(\Gamma \mathrm{x}_{\mathrm{n}}, \mathrm{x}_{\mathrm{n}}, \mathrm{x}_{\mathrm{n}}, \mathrm{t}\right)\right]$ by $(4)$

$\geq \beta_{\mathrm{n}}+\left(1-\beta_{\mathrm{n}}\right) G\left(\Gamma \mathrm{x}_{\mathrm{n}}, \mathrm{x}_{\mathrm{n}}, \mathrm{x}_{\mathrm{n}}, \mathrm{t}\right)$

M. Jeyaraman, R. D. Govt. Arts College, Sivagangai, Alagappa University, Thiruchirappalli, Tamil Nadu, India. (corresponding e-mail: jeya.math@ ${ }^{\text {gmail.com) }}$ 
$G\left(\Gamma \mathrm{x}_{\mathrm{n}}, \mathrm{x}_{\mathrm{n}}, \mathrm{x}_{\mathrm{n}}, \mathrm{t}\right) \geq G\left(\Gamma \mathrm{x}_{\mathrm{n}}, \Gamma \mathrm{y}_{\mathrm{n}}, \Gamma \mathrm{y}_{\mathrm{n}}, \mathrm{t} / 2\right) * G\left(\Gamma \mathrm{y}_{\mathrm{n}}, \mathrm{x}_{\mathrm{n}}, \mathrm{x}_{\mathrm{n}}, \mathrm{t} / 2\right)$ $\geq \beta_{\mathrm{n}}+\left(1-\beta_{\mathrm{n}}\right) G\left(\Gamma \mathrm{x}_{\mathrm{n}}, \mathrm{x}_{\mathrm{n}}, \mathrm{x}_{\mathrm{n}}, \mathrm{t} / 2 k\right)$.

It follows $G\left(\Gamma \mathrm{x}_{\mathrm{n}}, \mathrm{x}_{\mathrm{n}}, \mathrm{x}_{\mathrm{n}}, \mathrm{t}\right) \geq 1$ and $\mathrm{Tx}_{\mathrm{n}} \rightarrow \mathrm{x}$.

$G\left(\Gamma \mathrm{x}_{\mathrm{n}}, \mathrm{y}_{\mathrm{n}}, \mathrm{y}_{\mathrm{n}}, \mathrm{t}\right)=G\left(\Gamma \mathrm{x}, \triangle\left(\Gamma \mathrm{x}_{\mathrm{n}}, \mathrm{x}_{\mathrm{n}}, \beta_{\mathrm{n}}\right), \triangle\left(\Gamma \mathrm{x}_{\mathrm{n}}, \mathrm{x}_{\mathrm{n}}, \beta_{\mathrm{n}}\right), \mathrm{t}\right)$

$\geq \beta_{\mathrm{n}} G\left(\Gamma \mathrm{x}_{\mathrm{n}}, \Gamma \mathrm{x}, \Gamma \mathrm{x}, \mathrm{t}\right)+\left(1-\beta_{\mathrm{n}}\right) G\left(\mathrm{x}_{\mathrm{n}}, \Gamma \mathrm{x}, \Gamma \mathrm{x}, \mathrm{t}\right)$

$\geq \beta_{\mathrm{n}} G(\mathrm{x}, \Gamma \mathrm{x}, \Gamma \mathrm{x}, \mathrm{t})+\left(1-\beta_{\mathrm{n}}\right) G(\mathrm{x}, \Gamma \mathrm{x}, \Gamma \mathrm{x}, \mathrm{t})$.

It follows $G(\Gamma \mathrm{x}, \mathrm{x}, \mathrm{x}, \mathrm{t})=1$. Thus $\Gamma \mathrm{x}=\mathrm{x}$. Hence $\mathrm{x}$ is a fixed point of $\Gamma$.

\section{Theorem3.2}

Let $(\mathrm{X}, G, *)$ be a generalized fuzzy convex metric space and let $\Upsilon, \Gamma: \mathrm{X} \rightarrow \mathrm{X}$ be self mappings satisfying, $G(\Upsilon \mathrm{x}, \Gamma \mathrm{y}$, $\Gamma \mathrm{y}, k \mathrm{t}) \geq G(\Upsilon \mathrm{x}, \mathrm{y}, \mathrm{y}, \mathrm{t}) * G(\mathrm{x}, \Gamma \mathrm{y}, \Gamma \mathrm{y}, \mathrm{t})$, for all $\mathrm{x}, \mathrm{y} \in \mathrm{X}$ and $0<k<1$. Suppose that $\left\{\mathrm{x}_{\mathrm{n}}\right\}$ associated with $\mathrm{S}$ and $\Gamma$ is defined by:

$\mathrm{x}_{0} \in \mathrm{X}$

$\mathrm{x}_{\mathrm{n}+1}=\triangle\left(\Gamma \mathrm{y}_{\mathrm{n}}, \mathrm{x}_{\mathrm{n}}, \alpha_{\mathrm{n}}\right), \mathrm{n}=0,1,2 \ldots$

$\mathrm{y}_{\mathrm{n}}=\Delta\left(\Upsilon \mathrm{x}_{\mathrm{n}}, \mathrm{x}_{\mathrm{n}}, \beta_{\mathrm{n}}\right), \mathrm{n}=0,1,2 \ldots$

where $0 \leq \alpha_{\mathrm{n}}, \beta_{\mathrm{n}} \leq 1$ and $\beta_{\mathrm{n}}$ is bounded away from zero. If $\left\{x_{n}\right\}$ converges to some point $\mathrm{X} \in \mathrm{X}$ then $\mathrm{x}$ is the common fixed point of $\Gamma$ and $\Upsilon$.

\section{Proof}

Suppose $\mathrm{x}_{\mathrm{n}} \rightarrow \mathrm{x}$.

$G\left(\mathrm{x}_{\mathrm{n}}, \mathrm{x}_{\mathrm{n}+1}, \mathrm{x}_{\mathrm{n}+1}, \mathrm{t}\right)=G\left(\mathrm{x}_{\mathrm{n}}, \triangle\left(\Gamma \mathrm{y}_{\mathrm{n}}, \mathrm{x}_{\mathrm{n}}, \alpha_{\mathrm{n}}\right), \triangle\left(\Gamma \mathrm{y}_{\mathrm{n}}, \mathrm{x}_{\mathrm{n}}, \alpha_{\mathrm{n}}\right), \mathrm{t}\right)$

$\geq \alpha_{\mathrm{n}} G\left(\mathrm{x}_{\mathrm{n}}, \Gamma \mathrm{y}_{\mathrm{n}}, \Gamma \mathrm{y}_{\mathrm{n}}, \mathrm{t}\right)+\left(1-\alpha_{\mathrm{n}}\right) G\left(\mathrm{x}_{\mathrm{n}}, \mathrm{x}_{\mathrm{n}}, \mathrm{x}_{\mathrm{n}}, \mathrm{t}\right)$

$\geq \alpha_{\mathrm{n}} G\left(\mathrm{x}_{\mathrm{n}}, \Gamma \mathrm{y}_{\mathrm{n}}, \Gamma \mathrm{y}_{\mathrm{n}}, \mathrm{t}\right)+\left(1-\alpha_{\mathrm{n}}\right)$.

Therefore, $G\left(\mathrm{x}_{\mathrm{n}}, \Gamma \mathrm{y}_{\mathrm{n}}, \Gamma \mathrm{y}_{\mathrm{n}}, \mathrm{t}\right)=1$ and hence $\Gamma \mathrm{y}_{\mathrm{n}} \rightarrow \mathrm{x}$. Therefore, by (7) and (8),

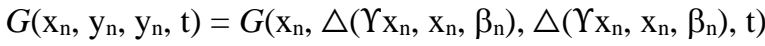

$\geq \beta_{\mathrm{n}} G\left(\mathrm{x}_{\mathrm{n}}, \Upsilon_{\mathrm{x}_{\mathrm{n}}}, \Upsilon_{\mathrm{x}_{\mathrm{n}}}, \mathrm{t}\right)+\left(1-\beta_{\mathrm{n}}\right) G\left(\mathrm{x}_{\mathrm{n}}, \mathrm{x}_{\mathrm{n}}, \mathrm{x}_{\mathrm{n}}, \mathrm{t}\right)$

$\geq \beta_{\mathrm{n}} G\left(\mathrm{x}_{\mathrm{n}}, \Upsilon_{\mathrm{x}_{\mathrm{n}}}, \Upsilon_{\mathrm{x}_{\mathrm{n}}}, \mathrm{t}\right)+\left(1-\beta_{\mathrm{n}}\right)$

$G\left(\Upsilon \mathrm{x}_{\mathrm{n}}, \mathrm{y}_{\mathrm{n}}, \mathrm{y}_{\mathrm{n}}, \mathrm{t}\right)=G\left(\Upsilon_{\mathrm{x}_{\mathrm{n}}}, \triangle\left(\Upsilon \mathrm{x}_{\mathrm{n}}, \mathrm{x}_{\mathrm{n}}, \beta_{\mathrm{n}}\right), \triangle\left(\Upsilon \mathrm{x}_{\mathrm{n}}, \mathrm{x}_{\mathrm{n}}, \beta_{\mathrm{n}}\right), \mathrm{t}\right)$

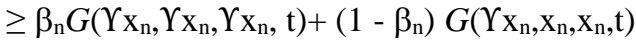

$\geq \beta_{\mathrm{n}}+\left(1-\beta_{\mathrm{n}}\right) G\left(\Upsilon_{\mathrm{x}_{\mathrm{n}}}, \mathrm{x}_{\mathrm{n}}, \mathrm{x}_{\mathrm{n}}, \mathrm{t}\right)$

$G\left(\Upsilon \mathrm{x}_{\mathrm{n}}, \Gamma \mathrm{y}_{\mathrm{n}}, \Gamma \mathrm{y}_{\mathrm{n}}, k \mathrm{t}\right) \geq G\left(\Upsilon \mathrm{x}_{\mathrm{n}}, \mathrm{y}_{\mathrm{n}}, \mathrm{y}_{\mathrm{n}}, \mathrm{t}\right) * G\left(\mathrm{x}_{\mathrm{n}}, \Gamma \mathrm{y}_{\mathrm{n}}, \Gamma \mathrm{y}_{\mathrm{n}}, \mathrm{t}\right)$

$\geq\left[\beta_{\mathrm{n}}+\left(1-\beta_{\mathrm{n}}\right) G\left(\Upsilon \mathrm{x}_{\mathrm{n}}, \mathrm{x}_{\mathrm{n}}, \mathrm{x}_{\mathrm{n}}, \mathrm{t}\right)\right] * 1$

$\geq \beta_{\mathrm{n}}+\left(1-\beta_{\mathrm{n}}\right) G\left(\Upsilon \mathrm{x}_{\mathrm{n}}, \mathrm{x}_{\mathrm{n}}, \mathrm{x}_{\mathrm{n}}, \mathrm{t}\right) \quad$ by $(10)$
$G\left(\Upsilon_{\mathrm{x}_{\mathrm{n}}}, \mathrm{x}_{\mathrm{n}}, \mathrm{x}_{\mathrm{n}}, \mathrm{t}\right) \geq G\left(\Upsilon_{\mathrm{x}_{\mathrm{n}}}, \Gamma \mathrm{y}_{\mathrm{n}}, \Gamma \mathrm{y}_{\mathrm{n}}, \mathrm{t} / 2\right) * G\left(\Gamma \mathrm{y}_{\mathrm{n}}, \mathrm{x}_{\mathrm{n}}, \mathrm{x}_{\mathrm{n}}, \mathrm{t} / 2\right)$

$\geq \beta_{\mathrm{n}} G\left(\mathrm{x}_{\mathrm{n}}, \Upsilon_{\mathrm{x}_{\mathrm{n}}}, \Upsilon_{\mathrm{x}_{\mathrm{n}}}, \mathrm{t} / 2 k\right) *\left(1-\beta_{\mathrm{n}}\right)$.

It follows $G\left(\Upsilon \mathrm{x}_{\mathrm{n}}, \mathrm{x}_{\mathrm{n}}, \mathrm{x}_{\mathrm{n}}, \mathrm{t}\right) \geq 1$ and $\Upsilon_{\mathrm{x}_{\mathrm{n}}} \rightarrow \mathrm{x}$. Also, $\mathrm{y}_{\mathrm{n}} \rightarrow \mathrm{x}$.

$G\left(\Gamma \mathrm{x}, \mathrm{y}_{\mathrm{n}}, \mathrm{y}_{\mathrm{n}}, \mathrm{t}\right)=G\left(\Gamma \mathrm{x}, \triangle\left(\Upsilon_{\mathrm{x}_{\mathrm{n}}}, \mathrm{x}_{\mathrm{n}}, \beta_{\mathrm{n}}\right), \triangle\left(\Upsilon_{\mathrm{x}_{\mathrm{n}}}, \mathrm{x}_{\mathrm{n}}, \beta_{\mathrm{n}}\right), \mathrm{t}\right)$

$\geq \beta_{\mathrm{n}} G\left(\Upsilon_{\mathrm{x}_{\mathrm{n}}}, \Gamma \mathrm{x}, \Gamma \mathrm{x}, \mathrm{t}\right)+\left(1-\beta_{\mathrm{n}}\right) G\left(\mathrm{x}_{\mathrm{n}}, \Gamma \mathrm{x}, \Gamma \mathrm{x}, \mathrm{t}\right)$

$\geq \beta_{\mathrm{n}} G(\mathrm{x}, \Gamma \mathrm{x}, \Gamma \mathrm{x}, \mathrm{t})+\left(1-\beta_{\mathrm{n}}\right) G(\mathrm{x}, \Gamma \mathrm{x}, \Gamma \mathrm{x}, \mathrm{t})$.

It follows $G(\Gamma \mathrm{x}, \mathrm{x}, \mathrm{x}, \mathrm{t})=1$. Thus $\Gamma \mathrm{x}=\mathrm{x}$.

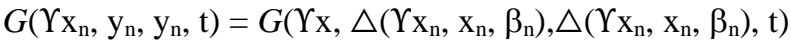

$\geq \beta_{\mathrm{n}} G\left(\Upsilon \mathrm{x}, \Upsilon_{\mathrm{x}}, \Upsilon_{\mathrm{x}}, \mathrm{t}\right)+\left(1-\beta_{\mathrm{n}}\right) G\left(\Upsilon \mathrm{x}, \mathrm{x}_{\mathrm{n}}, \mathrm{x}_{\mathrm{n}}, \mathrm{t}\right)$

$\geq \beta_{\mathrm{n}} G(\Upsilon \mathrm{x}, \mathrm{x}, \mathrm{x}, \mathrm{t})+\left(1-\beta_{\mathrm{n}}\right) G(\Upsilon \mathrm{x}, \mathrm{x}, \mathrm{x}, \mathrm{t})$.

Thus, $\Gamma x=\Upsilon x=x$. Hence $x$ is the common fixed point of $\Gamma$ and $Y$

E. Theorem 3.3

Let $(\mathrm{X}, G, *)$ be a generalized fuzzy convex metric space and let $\Upsilon, \Gamma: X \rightarrow X$ be self mappings satisfying, $G(\Upsilon \mathrm{x}, \Gamma \mathrm{y}, \Gamma \mathrm{y}, k \mathrm{t}) \geq G(\mathrm{x}, \mathrm{y}, \mathrm{y}, \mathrm{t})$, for all $\mathrm{x}, \mathrm{y} \in \mathrm{X}$ and $0<k<$ 1. Suppose that $\left\{\mathrm{x}_{\mathrm{n}}\right\}$ associated with $\Upsilon$ and $\Gamma$ is defined by:

$\mathrm{x}_{0} \in \mathrm{X}$

$\mathrm{x}_{\mathrm{n}+1}=\triangle\left(\Gamma \mathrm{y}_{\mathrm{n}}, \mathrm{x}_{\mathrm{n}}, \alpha_{\mathrm{n}}\right), \mathrm{n}=0,1,2 \ldots$

$\mathrm{y}_{\mathrm{n}}=\Delta\left(\Upsilon \mathrm{x}_{\mathrm{n}}, \mathrm{x}_{\mathrm{n}}, \beta_{\mathrm{n}}\right), \mathrm{n}=0,1,2 \ldots$

where $0 \leq \alpha_{n}, \beta_{n} \leq 1$ and $\beta_{n}$ is bounded away from zero.

If $\left\{x_{n}\right\}$ converges to some point $x \in X$ then $x$ is the common fixed point of $\Gamma$ and $\Upsilon$.

\section{F. Proof}

Suppose $\mathrm{x}_{\mathrm{n}} \rightarrow \mathrm{X}$.

$G\left(\mathrm{x}_{\mathrm{n}}, \mathrm{x}_{\mathrm{n}+1}, \mathrm{x}_{\mathrm{n}+1}, \mathrm{t}\right)=G\left(\mathrm{x}_{\mathrm{n}}, \triangle\left(\Gamma \mathrm{y}_{\mathrm{n}}, \mathrm{x}_{\mathrm{n}}, \alpha_{\mathrm{n}}\right), \triangle\left(\Gamma \mathrm{y}_{\mathrm{n}}, \mathrm{x}_{\mathrm{n}}, \alpha_{\mathrm{n}}\right), \mathrm{t}\right)$

$\geq \alpha_{\mathrm{n}} G\left(\mathrm{x}_{\mathrm{n}}, \Gamma \mathrm{y}_{\mathrm{n}}, \Gamma \mathrm{y}_{\mathrm{n}}, \mathrm{t}\right)+\left(1-\alpha_{\mathrm{n}}\right)$

$\geq \alpha_{\mathrm{n}} G\left(\mathrm{x}_{\mathrm{n}}, \Gamma \mathrm{y}_{\mathrm{n}}, \Gamma \mathrm{y}_{\mathrm{n}}, \mathrm{t}\right)+\left(1-\alpha_{\mathrm{n}}\right)$.

Therefore, $G\left(\mathrm{x}_{\mathrm{n}}, \Gamma \mathrm{y}_{\mathrm{n}}, \Gamma \mathrm{y}_{\mathrm{n}}, \mathrm{t}\right)=1$ and hence $\Gamma \mathrm{y}_{\mathrm{n}} \rightarrow \mathrm{x}$.

$G\left(\mathrm{x}_{\mathrm{n}}, \mathrm{y}_{\mathrm{n}}, \mathrm{y}_{\mathrm{n}}, \mathrm{t}\right)=G\left(\mathrm{x}_{\mathrm{n}}, \triangle\left(\mathrm{x}_{\mathrm{n}}, \mathrm{x}_{\mathrm{n}}, \beta_{\mathrm{n}}\right), \triangle\left(\Upsilon_{\mathrm{x}}, \mathrm{x}_{\mathrm{n}}, \beta_{\mathrm{n}}\right), \mathrm{t}\right)$

$\geq \beta_{\mathrm{n}} G\left(\mathrm{x}_{\mathrm{n}}, \Upsilon_{\mathrm{x}_{\mathrm{n}}}, \Upsilon_{\mathrm{x}_{\mathrm{n}}}, \mathrm{t}\right)+\left(1-\beta_{\mathrm{n}}\right) G\left(\mathrm{x}_{\mathrm{n}}, \mathrm{x}_{\mathrm{n}}, \mathrm{x}_{\mathrm{n}}, \mathrm{t}\right)$

$\geq \beta_{\mathrm{n}} G\left(\mathrm{x}_{\mathrm{n}}, \Upsilon \mathrm{x}_{\mathrm{n}}, \Upsilon \mathrm{x}_{\mathrm{n}}, \mathrm{t}\right)+\left(1-\beta_{\mathrm{n}}\right)$

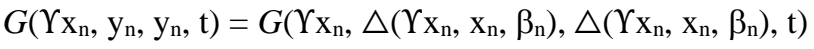

$\geq \beta_{\mathrm{n}} G\left(\Upsilon_{\mathrm{x}_{\mathrm{n}}}, \Upsilon_{\mathrm{x}_{\mathrm{n}}}, \Upsilon \mathrm{x}_{\mathrm{n}}, \mathrm{t}\right)+\left(1-\beta_{\mathrm{n}}\right) G\left(\Upsilon \mathrm{x}_{\mathrm{n}}, \mathrm{x}_{\mathrm{n}}, \mathrm{x}_{\mathrm{n}}, \mathrm{t}\right)$ 
$\geq \beta_{\mathrm{n}}+\left(1-\beta_{\mathrm{n}}\right) G\left(\Upsilon \mathrm{x}_{\mathrm{n}}, \mathrm{x}_{\mathrm{n}}, \mathrm{x}_{\mathrm{n}}, \mathrm{t}\right)$.

$G\left(\Upsilon_{\mathrm{x}_{\mathrm{n}}}, \Gamma \mathrm{y}_{\mathrm{n}}, \Gamma \mathrm{y}_{\mathrm{n}}, k \mathrm{t}\right) \geq G\left(\mathrm{x}_{\mathrm{n}}, \mathrm{y}_{\mathrm{n}}, \mathrm{y}_{\mathrm{n}}, \mathrm{t}\right)$

$\geq \beta_{\mathrm{n}} G\left(\mathrm{x}_{\mathrm{n}}, \Upsilon \mathrm{x}_{\mathrm{n}}, \Upsilon \mathrm{x}_{\mathrm{n}}, \mathrm{t}\right)+\left(1-\beta_{\mathrm{n}}\right) \quad$ by $(15)$

$G\left(\Upsilon_{\mathrm{x}_{\mathrm{n}}}, \mathrm{x}_{\mathrm{n}}, \mathrm{x}_{\mathrm{n}}, \mathrm{t}\right) \geq G\left(\Upsilon_{\mathrm{x}_{\mathrm{n}}}, \Gamma \mathrm{y}_{\mathrm{n}}, \Gamma \mathrm{y}_{\mathrm{n}}, \mathrm{t} / 2\right) * G\left(\mathrm{Ty}_{\mathrm{n}}, \mathrm{x}_{\mathrm{n}}, \mathrm{x}_{\mathrm{n}}, \mathrm{t} / 2\right)$

$\geq \beta_{\mathrm{n}} G\left(\mathrm{x}_{\mathrm{n}}, \Upsilon \mathrm{x}_{\mathrm{n}}, \Upsilon_{\mathrm{x}_{\mathrm{n}}}, \mathrm{t} / 2 k\right)+\left(1-\beta_{\mathrm{n}}\right)$.

It follows $G\left(\Upsilon \mathrm{x}_{\mathrm{n}}, \mathrm{x}_{\mathrm{n}}, \mathrm{x}_{\mathrm{n}}, \mathrm{t}\right) \geq 1$ and $\Upsilon \mathrm{x}_{\mathrm{n}} \rightarrow \mathrm{x}$. Also $\mathrm{y}_{\mathrm{n}} \rightarrow \mathrm{x}$. Now, $G\left(\Gamma \mathrm{x}, \mathrm{y}_{\mathrm{n}}, \mathrm{y}_{\mathrm{n}}, \mathrm{t}\right)=G\left(\Gamma \mathrm{x}, \triangle\left(\Upsilon_{\mathrm{x}}, \mathrm{x}_{\mathrm{n}}, \beta_{\mathrm{n}}\right), \triangle\left(\Upsilon_{\mathrm{x}}, \mathrm{x}_{\mathrm{n}}, \beta_{\mathrm{n}}\right), \mathrm{t}\right)$

$\geq \beta_{\mathrm{n}} G\left(\Upsilon \mathrm{x}_{\mathrm{n}}, \Gamma \mathrm{x}, \Gamma \mathrm{x}, \mathrm{t}\right)+\left(1-\beta_{\mathrm{n}}\right) G\left(\mathrm{x}_{\mathrm{n}}, \Gamma \mathrm{x}, \Gamma \mathrm{x}, \mathrm{t}\right)$

$\geq \beta_{\mathrm{n}} G(\mathrm{x}, \Gamma \mathrm{x}, \Gamma \mathrm{x}, \mathrm{t})+\left(1-\beta_{\mathrm{n}}\right) G(\mathrm{x}, \Gamma \mathrm{x}, \Gamma \mathrm{x}, \mathrm{t})$.

It follows $G(\Gamma \mathrm{x}, \mathrm{x}, \mathrm{x}, \mathrm{t})=1$. Thus $\Gamma \mathrm{x}=\mathrm{x}$.

$G\left(\Upsilon \mathrm{x}, \mathrm{y}_{\mathrm{n}}, \mathrm{y}_{\mathrm{n}}, \mathrm{t}\right)=G\left(\Upsilon \mathrm{x}, \Delta\left(\Upsilon_{\mathrm{n}}, \mathrm{x}_{\mathrm{n}}, \beta_{\mathrm{n}}\right), \Delta\left(\Upsilon_{\mathrm{x}_{\mathrm{n}}}, \mathrm{x}_{\mathrm{n}}, \beta_{\mathrm{n}}\right), \mathrm{t}\right)$

$\geq \beta_{\mathrm{n}} G\left(\Upsilon \mathrm{x}, \Upsilon_{\mathrm{x}_{\mathrm{n}}}, \Upsilon_{\mathrm{x}_{\mathrm{n}}}, \mathrm{t}\right)+\left(1-\beta_{\mathrm{n}}\right) G\left(\Upsilon \mathrm{x}, \mathrm{x}_{\mathrm{n}}, \mathrm{x}_{\mathrm{n}}, \mathrm{t}\right)$

$\geq \beta_{\mathrm{n}} G(\Upsilon \mathrm{x}, \mathrm{x}, \mathrm{x}, \mathrm{t})+\left(1-\beta_{\mathrm{n}}\right) G(\Upsilon \mathrm{x}, \mathrm{x}, \mathrm{x}, \mathrm{t})$.

Thus, $\Gamma x=\Upsilon x=x$. Hence $x$ is the common fixed point of $\Gamma$ and $\Upsilon$

\section{G. Theorem3.4}

Let $(\mathrm{X}, G, *)$ be a generalized fuzzy convex metric space and let $\Upsilon, \Gamma: \mathrm{X} \rightarrow \mathrm{X}$ be self mappings satisfying, $G(\Gamma \mathrm{x}, \Gamma \mathrm{y}$, $\Gamma \mathrm{y}, k \mathrm{t}) \geq G(\Upsilon \mathrm{x}, \Upsilon \mathrm{y}, \Upsilon \mathrm{y}, \mathrm{t})$, for all $\mathrm{x}, \mathrm{y} \in \mathrm{X}$ and $0<k<1$. Suppose that $\left\{\mathrm{x}_{\mathrm{n}}\right\}$ associated with $\Upsilon$ and $\Gamma$ is defined by

$\mathrm{x}_{0} \in \mathrm{X}$

$\mathrm{x}_{\mathrm{n}+1}=\Delta\left(\Gamma \mathrm{y}_{\mathrm{n}}, \mathrm{x}_{\mathrm{n}}, \alpha_{\mathrm{n}}\right), \mathrm{n}=0,1,2 \ldots$

$\mathrm{y}_{\mathrm{n}}=\triangle\left(\Upsilon \mathrm{x}_{\mathrm{n}}, \mathrm{x}_{\mathrm{n}}, \beta_{\mathrm{n}}\right), \mathrm{n}=0,1,2 \ldots$

where $0 \leq \alpha_{\mathrm{n}}, \beta_{\mathrm{n}} \leq 1$ and $\beta_{\mathrm{n}}$ is bounded away from zero. If $\left\{x_{n}\right\}$ converges to some point $\mathrm{x} \in \mathrm{X}$ then $\mathrm{x}$ is the common fixed point of $\Gamma$ and $\Upsilon$.

\section{H. Proof}

Suppose $\mathrm{x}_{\mathrm{n}} \rightarrow \mathrm{X}$.

$G\left(\mathrm{x}_{\mathrm{n}}, \mathrm{x}_{\mathrm{n}+1}, \mathrm{x}_{\mathrm{n}+1}, \mathrm{t}\right)=G\left(\mathrm{x}_{\mathrm{n}}, \triangle\left(\Gamma \mathrm{y}_{\mathrm{n}}, \mathrm{x}_{\mathrm{n}}, \alpha_{\mathrm{n}}\right), \triangle\left(\Gamma \mathrm{y}_{\mathrm{n}}, \mathrm{x}_{\mathrm{n}}, \alpha_{\mathrm{n}}\right), \mathrm{t}\right)$

$\geq \alpha_{\mathrm{n}} G\left(\mathrm{x}_{\mathrm{n}}, \Gamma \mathrm{y}_{\mathrm{n}}, \Gamma \mathrm{y}_{\mathrm{n}}, \mathrm{t}\right)+\left(1-\alpha_{\mathrm{n}}\right) G\left(\mathrm{x}_{\mathrm{n}}, \mathrm{x}_{\mathrm{n}}, \mathrm{x}_{\mathrm{n}}, \mathrm{t}\right)$

$\geq \alpha_{\mathrm{n}} G\left(\mathrm{x}_{\mathrm{n}}, \Gamma \mathrm{y}_{\mathrm{n}}, \Gamma \mathrm{y}_{\mathrm{n}}, \mathrm{t}\right)+\left(1-\alpha_{\mathrm{n}}\right)$.

Therefore, $G\left(\mathrm{x}_{\mathrm{n}}, \Gamma \mathrm{y}_{\mathrm{n}}, \Gamma \mathrm{y}_{\mathrm{n}}, \mathrm{t}\right)=1$ and hence $\Gamma \mathrm{y}_{\mathrm{n}} \rightarrow \mathrm{x}$.

$G\left(\mathrm{x}_{\mathrm{n}}, \mathrm{y}_{\mathrm{n}}, \mathrm{y}_{\mathrm{n}}, \mathrm{t}\right)=G\left(\mathrm{x}_{\mathrm{n}}, \triangle\left(\Upsilon \mathrm{x}_{\mathrm{n}}, \mathrm{x}_{\mathrm{n}}, \beta_{\mathrm{n}}\right), \triangle\left(\Upsilon_{\mathrm{x}}, \mathrm{x}_{\mathrm{n}}, \beta_{\mathrm{n}}\right), \mathrm{t}\right)$

$\geq \beta_{\mathrm{n}} G\left(\mathrm{x}_{\mathrm{n}}, \Upsilon_{\mathrm{x}_{\mathrm{n}}}, \Upsilon \mathrm{x}_{\mathrm{n}}, \mathrm{t}\right)+\left(1-\beta_{\mathrm{n}}\right) G\left(\mathrm{x}_{\mathrm{n}}, \mathrm{x}_{\mathrm{n}}, \mathrm{x}_{\mathrm{n}}, \mathrm{t}\right)$

$\geq \beta_{\mathrm{n}} G\left(\mathrm{x}_{\mathrm{n}}, \Upsilon \mathrm{x}_{\mathrm{n}}, \Upsilon \mathrm{x}_{\mathrm{n}}, \mathrm{t}\right)+\left(1-\beta_{\mathrm{n}}\right)$ and

$G\left(\Upsilon \mathrm{x}_{\mathrm{n}}, \mathrm{y}_{\mathrm{n}}, \mathrm{y}_{\mathrm{n}}, \mathrm{t}\right)=G\left(\Upsilon \mathrm{x}_{\mathrm{n}}, \Delta\left(\Upsilon \mathrm{x}_{\mathrm{n}}, \mathrm{x}_{\mathrm{n}}, \beta_{\mathrm{n}}\right), \Delta\left(\Upsilon \mathrm{x}_{\mathrm{n}}, \mathrm{x}_{\mathrm{n}}, \beta_{\mathrm{n}}\right), \mathrm{t}\right)$

$\geq \beta_{\mathrm{n}} G\left(\Upsilon \mathrm{x}_{\mathrm{n}}, \Upsilon \mathrm{x}_{\mathrm{n}}, \Upsilon \mathrm{x}_{\mathrm{n}}, \mathrm{t}\right)+\left(1-\beta_{\mathrm{n}}\right) G\left(\Upsilon \mathrm{x}_{\mathrm{n}}, \mathrm{x}_{\mathrm{n}}, \mathrm{x}_{\mathrm{n}}, \mathrm{t}\right)$ $\geq \beta_{\mathrm{n}}+\left(1-\beta_{\mathrm{n}}\right) G\left(\Upsilon \mathrm{x}_{\mathrm{n}}, \mathrm{x}_{\mathrm{n}}, \mathrm{x}_{\mathrm{n}}, \mathrm{t}\right)$.

$G\left(\Upsilon \mathrm{x}_{\mathrm{n}}, \Gamma \mathrm{y}_{\mathrm{n}}, \Gamma \mathrm{y}_{\mathrm{n}}, k \mathrm{t}\right) \geq G\left(\mathrm{x}_{\mathrm{n}}, \mathrm{y}_{\mathrm{n}}, \mathrm{y}_{\mathrm{n}}, \mathrm{t}\right)$

$\geq \beta_{\mathrm{n}} G\left(\mathrm{x}_{\mathrm{n}}, \Upsilon \mathrm{x}_{\mathrm{n}}, \Upsilon \mathrm{x}_{\mathrm{n}}, \mathrm{t}\right)+\left(1-\beta_{\mathrm{n}}\right) \quad$ by $(20)$

$G\left(\Upsilon \mathrm{x}_{\mathrm{n}}, \mathrm{x}_{\mathrm{n}}, \mathrm{x}_{\mathrm{n}}, \mathrm{t}\right) \geq G\left(\Upsilon \mathrm{x}_{\mathrm{n}}, \Gamma \mathrm{y}_{\mathrm{n}}, \Gamma \mathrm{y}_{\mathrm{n}}, \mathrm{t} / 2\right) * G\left(\Gamma \mathrm{y}_{\mathrm{n}}, \mathrm{x}_{\mathrm{n}}, \mathrm{x}_{\mathrm{n}}, \mathrm{t} / 2\right)$

$\geq \beta_{\mathrm{n}} G\left(\mathrm{x}_{\mathrm{n}}, \Upsilon \mathrm{x}_{\mathrm{n}}, \Upsilon \mathrm{x}_{\mathrm{n}}, \mathrm{t} / 2 k\right)+\left(1-\beta_{\mathrm{n}}\right)$.

It follows $G\left(\Upsilon_{\mathrm{x}_{\mathrm{n}}}, \mathrm{x}_{\mathrm{n}}, \mathrm{x}_{\mathrm{n}}, \mathrm{t}\right) \geq 1$ and $\Upsilon_{\mathrm{x}_{\mathrm{n}}} \rightarrow \mathrm{x}$. Also, $\mathrm{y}_{\mathrm{n}} \rightarrow \mathrm{x}$.

$G\left(\Gamma \mathrm{x}, \mathrm{y}_{\mathrm{n}}, \mathrm{y}_{\mathrm{n}}, \mathrm{t}\right)=G\left(\Gamma \mathrm{x}, \triangle\left(\Upsilon_{\mathrm{x}_{\mathrm{n}}}, \mathrm{x}_{\mathrm{n}}, \beta_{\mathrm{n}}\right), \triangle\left(\Upsilon_{\mathrm{x}}, \mathrm{x}_{\mathrm{n}}, \beta_{\mathrm{n}}\right), \mathrm{t}\right)$

$\geq \beta_{\mathrm{n}} G\left(\Upsilon \mathrm{x}_{\mathrm{n}}, \Gamma \mathrm{x}, \Gamma \mathrm{x}, \mathrm{t}\right)+\left(1-\beta_{\mathrm{n}}\right) G\left(\mathrm{x}_{\mathrm{n}}, \Gamma \mathrm{x}, \Gamma \mathrm{x}, \mathrm{t}\right)$

$\geq \beta_{\mathrm{n}} G(\mathrm{x}, \Gamma \mathrm{x}, \Gamma \mathrm{x}, \mathrm{t})+\left(1-\beta_{\mathrm{n}}\right) G(\mathrm{x}, \Gamma \mathrm{x}, \Gamma \mathrm{x}, \mathrm{t})$.

It follows $G(\Gamma \mathrm{x}, \mathrm{x}, \mathrm{x}, \mathrm{t})=1$. Thus $\Gamma \mathrm{x}=\mathrm{x}$.

$G\left(\Upsilon \mathrm{x}, \mathrm{y}_{\mathrm{n}}, \mathrm{y}_{\mathrm{n}}, \mathrm{t}\right)=G\left(\Upsilon \mathrm{x}, \Delta\left(\Upsilon_{\mathrm{x}_{\mathrm{n}}}, \mathrm{x}_{\mathrm{n}}, \beta_{\mathrm{n}}\right), \Delta\left(\Upsilon_{\mathrm{x}_{\mathrm{n}}}, \mathrm{x}_{\mathrm{n}}, \beta_{\mathrm{n}}\right), \mathrm{t}\right)$

$\geq \beta_{\mathrm{n}} G\left(\Upsilon \mathrm{x}, \Upsilon_{\mathrm{x}_{\mathrm{n}}}, \Upsilon_{\mathrm{x}_{\mathrm{n}}}, \mathrm{t}\right)+\left(1-\beta_{\mathrm{n}}\right) G\left(\Upsilon \mathrm{x}, \mathrm{x}_{\mathrm{n}}, \mathrm{x}_{\mathrm{n}}, \mathrm{t}\right)$

$\geq \beta_{\mathrm{n}} G(\Upsilon \mathrm{x}, \mathrm{x}, \mathrm{x}, \mathrm{t})+\left(1-\beta_{\mathrm{n}}\right) G(\Upsilon \mathrm{x}, \mathrm{x}, \mathrm{x}, \mathrm{t})$.

Thus, $\Gamma x=\Upsilon x=x$. Hence $x$ is the common fixed point of $\Gamma$ and $\Upsilon$.

\section{Definition 3.5}

Let $(\mathrm{X}, G, *)$ be a complete generalized fuzzy convex metric space and let $\Gamma: X \rightarrow X$ be continuous mapping $\Gamma$ is a quasi-non expansive if $G(\Gamma \mathrm{x}, \mathrm{x}, \mathrm{x}, \mathrm{t}) \geq G(\mathrm{x}, \mathrm{x}, \mathrm{x}, \mathrm{t})$, for all $\mathrm{x}$ $\in X$ and $\mathrm{x}$ is a fixed point of $\Gamma$.

\section{J. Theorem 3.6}

Let $(\mathrm{X}, G, *)$ be a complete generalized fuzzy convex metric space and let $\Upsilon, \Gamma: X \rightarrow X$ be quasi-non expansive mappings. Suppose that $\left\{x_{n}\right\}$ associated with $\Upsilon$ and $\Gamma$ is defined by:

$\mathrm{x}_{0} \in \mathrm{X}$

$\mathrm{x}_{\mathrm{n}+1}=\Delta\left(\Gamma \mathrm{y}_{\mathrm{n}}, \mathrm{x}_{\mathrm{n}}, \alpha_{\mathrm{n}}\right), \mathrm{n}=0,1,2 \ldots$

$\mathrm{y}_{\mathrm{n}}=\triangle\left(\Upsilon \mathrm{x}_{\mathrm{n}}, \mathrm{x}_{\mathrm{n}}, \beta_{\mathrm{n}}\right), \mathrm{n}=0,1,2 \ldots$

where $0 \leq \alpha_{\mathrm{n}}, \beta_{\mathrm{n}} \leq 1$ and $\left\{\alpha_{\mathrm{n}}\right\}$ is bounded away from zero. If $\left\{\mathrm{x}_{\mathrm{n}}\right\}$ converges to some point $\mathrm{x} \in \mathrm{X}$, then $\mathrm{x}$ is the common fixed point of $\Gamma$ and $\Upsilon$.

K. Proof

Let $\mathrm{x} \in \mathrm{F}(\Gamma) \cap \mathrm{F}(\Upsilon)$. Then:

$G\left(\mathrm{x}_{\mathrm{n}+1}, \mathrm{x}, \mathrm{x}, \mathrm{t}\right)=G\left(\triangle\left(\Gamma \mathrm{y}_{\mathrm{n}}, \mathrm{x}_{\mathrm{n}}, \alpha_{\mathrm{n}}\right), \mathrm{x}, \mathrm{x}, \mathrm{t}\right)$

$\geq \alpha_{\mathrm{n}} G\left(\Gamma \mathrm{y}_{\mathrm{n}}, \mathrm{x}, \mathrm{x}, \mathrm{t}\right)+\left(1-\alpha_{\mathrm{n}}\right) G\left(\mathrm{x}_{\mathrm{n}}, \mathrm{x}, \mathrm{x}, \mathrm{t}\right)$

$\geq \alpha_{\mathrm{n}} G\left(\mathrm{y}_{\mathrm{n}}, \mathrm{x}, \mathrm{x}, \mathrm{t}\right)+\left(1-\alpha_{\mathrm{n}}\right) G\left(\mathrm{x}_{\mathrm{n}}, \mathrm{x}, \mathrm{x}, \mathrm{t}\right)$

$\geq \alpha_{\mathrm{n}} G\left(\triangle\left(\Upsilon \mathrm{x}_{\mathrm{n}}, \mathrm{x}_{\mathrm{n}}, \beta_{\mathrm{n}}\right), \mathrm{x}, \mathrm{x}, \mathrm{t}\right)+\left(1-\alpha_{\mathrm{n}}\right) G\left(\mathrm{x}_{\mathrm{n}}, \mathrm{x}, \mathrm{x}, \mathrm{t}\right)$ 
$\geq \alpha_{\mathrm{n}} \beta_{\mathrm{n}} G\left(\Upsilon_{\mathrm{x}}, \mathrm{x}, \mathrm{x}, \mathrm{t}\right)+\alpha_{\mathrm{n}}\left(1-\beta_{\mathrm{n}}\right) G\left(\mathrm{x}_{\mathrm{n}}, \mathrm{x}, \mathrm{x}, \mathrm{t}\right)$

$$
+\left(1-\alpha_{\mathrm{n}}\right) G\left(\mathrm{x}_{\mathrm{n}}, \mathrm{x}, \mathrm{x}, \mathrm{t}\right)
$$

$\geq\left[\alpha_{n} \beta_{n}+\alpha_{n}\left(1-\beta_{n}\right)+\left(1-\alpha_{n}\right)\right] G\left(x_{n}, x, x, t\right)$

$\geq G\left(\mathrm{x}_{\mathrm{n}}, \mathrm{x}, \mathrm{x}, \mathrm{t}\right)$.

Thus, $G\left(\mathrm{x}_{\mathrm{n}+1}, \mathrm{x}, \mathrm{x}, \mathrm{t}\right) \geq G\left(\mathrm{x}_{\mathrm{n}}, \mathrm{x}, \mathrm{x}, \mathrm{t}\right) \geq G\left(\mathrm{x}_{\mathrm{n}-1}, \mathrm{x}, \mathrm{x}, \mathrm{t}\right) \geq \ldots$ This implies $G\left(\mathrm{x}_{\mathrm{n}}, \mathrm{x}, \mathrm{x}, \mathrm{t}\right) \geq 1$. Hence $\left\{\mathrm{x}_{\mathrm{n}}\right\}$ converges to $\mathrm{x}$. The following result is a special case of the above theorem.

\section{Corollary 3.7}

Let $(\mathrm{X}, G, *)$ be a complete generalized fuzzy convex metric space and let $\Gamma: \mathrm{X} \rightarrow \mathrm{X}$ be quasi-non expansive mappings. The sequence $\left\{x_{n}\right\}$ is defined by:

$\mathrm{x}_{0} \in \mathrm{X}$

and

$\mathrm{x}_{\mathrm{n}+1}=\Delta\left(\Gamma \mathrm{y}_{\mathrm{n}}, \mathrm{x}_{\mathrm{n}}, \alpha_{\mathrm{n}}\right)$

$\mathrm{y}_{\mathrm{n}}=\triangle\left(\Gamma \mathrm{x}_{\mathrm{n}}, \mathrm{x}_{\mathrm{n}}, \beta_{\mathrm{n}}\right), \mathrm{n}=0,1,2, \ldots$

where $0 \leq \alpha_{\mathrm{n}}, \beta_{\mathrm{n}} \leq 1$. Then $\left\{\mathrm{x}_{\mathrm{n}}\right\}$ converges to the fixed point of $\Gamma$.

\section{Proof}

The proof of the corollary immediately follows by putting $\Upsilon=\Gamma$ in the previous theorem.

\section{CONCLUSION}

In this paper, we have explored the concept of convex structure in generalized fuzzy metric spaces and proved common fixed point theorems for a pair of self-mappings under sufficient contractive type conditions.

\section{REFERENCES}

[1] George. A. and Veeramani (1997), Fuzzy sets and Systems, 365-368.

[2] Jungck. G, (1986), Compatible mappings band common fixed points Internat, J. Math.and Math. Sci.9, 771-779.

[3] Kaleva. O and Seikkala. S, (1984), On fuzzy metric spaces, Fuzzy sets and systems, 12, 215-229.

[4] Kramosil. I and Michalek. J, (1975), Fuzzy metric and statistical metric spaces, Kubernetika, 11, 336-344.

[5] Kubiaczyk. I and Sushil Sharma, (2002), Common fixed point multi maps in fuzzy metric space, East Asian Math. J. 18, (2), 175-182.

[6] Mishra. S. N, Sharma. N and Singh. S. L, (1994), Common fixed points of maps on fuzzy metric spaces, Internat. J. Math.and Math. BSci.,17,253-258.

[7] Singh. B and Jain. S, (2007), Generalized theorem in fuzzy metric spaces, Southeast Asian Bulletin of Mathematics, 31, 1-16.

[8] Sharma. S, (2002), On fuzzy metric space, Southeast Asian Bulletin of Mathematics, 26, 133-145.

[9] Sushil Sharma, (2002), Common fixed point theorems in fuzzy metric spaces, Fuzzy Setsand Systems, 127, 345-352.

[10] Thanithamil. A. and Thirunavukarasu. P, (2014), Some Results in fuzzy metric spaces with convex structure, Int. Journal of Math .Analysis, 8 , 57, 2827-2836.

[11] Vijayaraju. P and Sajath. Z.M.I, (2009), Some common fixed point theorems in fuzzy metric spaces, Int. Journal of Math.Analysis,3, 701710 .

[12] Zadeh, L. A, (1965), Fuzzy sets, Information and Computation, 8, 338353.

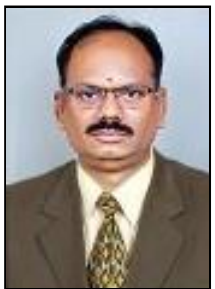

Dr. M. Jeyaraman having more than 20 years of teaching experience and 10 years of research experience, is working as Assistant Professor in PG and Research Department of Mathematics, Raja Doraisingam Government Arts College, Sivagangai, Tamil Nadu in India. He received his Ph.D. degree in 2010. Also, he has M.Phil (Mathematics), M.Ed. He has published several articles in collaboration with profound scholarly professors of various countries and his immense contribution in reputed journals of national and international is more than one hundred twenty five papers. He has presented more than ten research papers in national and international conferences. Five research scholars were awarded Ph.D degree and thirty sixresearch scholars were awarded an M.Phil degree in Mathematics. He is also reviewer of many prestigious professional bodies like Mathematical Reviews etc. His research interests are Fixed Point Theory, Fuzzy Set Theory, Functional Analysis, Topology and Differential Equations.

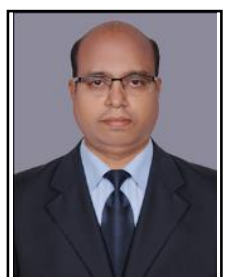

V. Pazhani doing Ph.D., under the guidance of Dr. V. Vinoba at Bharathidasan University, Tiruchirappalli. He has more than 20 years of teaching experience, presently working as Assistant Professor in PG and Research Department of Mathematics, Raja Doraisingam Government Arts College, Sivagangai, Tamil Nadu in India. He has presented 05 research papers in national and international conferences and published more than 10 research articles in reputed international journals. His research interests are Fixed Point Theory, Fuzzy Theory. 